\title{
Una Desigualdad de Carleman para un sistema de Ecuaciones Parabólicas Acopladas
}

\author{
Victor Rafael Cabanillas Zannini ${ }^{1}$
}

Resumen: En este trabajo consideramos un sistema linealizado de ecuaciones parabólicas acopladas asociadas a un sistema semilineal de ecuaciones del calor. Siguiendo las ideas de O. Imanuvilov, M. Yamamoto y L. de Teresa probamos una estimativa de tipo Carleman para un sistema de ecuaciones parabólicas que envuelven términos gradientes. Esta clase de sistemas aparecen en el estudio de controles que hacen insensitiva a la norma de la solución de una ecuación semilineal del calor.

Palabras clave: Desigualdad de Carleman, controles insensitivos, desigualdad de observabilidad.

\section{A Carleman's Inequality for a System of Coupled Parabolic Equations}

\begin{abstract}
In this work we consider a linearized system of parabolic equations associated to a semilinear system of heat equations. Following the ideas of $\mathrm{O}$. Imanuvilov, M. Yamamoto and L. de Teresa we prove a estimate of Carleman type for a system of parabolic equations involving gradient terms. This class of systems appears in the treatment of controls insensitizing the norm of the solution of a semilinear heat equation.
\end{abstract}

Key words: Carleman inequality, insensitizing controls, observability inequality.

\section{Introduction}

Sea $\Omega$ un dominio abierto y acotado de $\mathbb{R}^{n}, n \geq 1$, con frontera suficientemente regular $\Gamma$. Sea $T>0$, y denotemos por $Q=\Omega \times(0, T)$ el cilindro generado por $\Omega$ y por $\Sigma=\Gamma \times(0, T)$ su frontera lateral. Consideremos el siguiente sistema de ecuaciones parabólicas

$$
\begin{gathered}
\begin{cases}p^{\prime}-\Delta p+A p+B \cdot \nabla p=0 & , \text { en } Q \\
p=0 & , \text { sobre } \Sigma \\
p(0)=p^{0} & , \text { en } \Omega,\end{cases} \\
\begin{cases}-z^{\prime}-\Delta z+a z-\operatorname{div}(b z)=p 1_{0} & , \text { en } Q \\
z=0 & , \text { sobre } \Sigma \\
z(T)=0 & , \text { en } \Omega .\end{cases}
\end{gathered}
$$

donde $a, A \in L^{\infty}(Q), b, B \in\left[L^{\infty}(Q)\right]^{n}$, y $p^{0} \in L^{2}(\Omega)$.

Es bien conocido que bajo las hipótesis anteriores, el sistema (1.1)-(1.2) posee una única solución $(p, z)$ tal que

$$
(p, z) \in\left[C\left([0, T]: L^{2}\right) \cap L^{2}\left(0, T ; H_{0}^{1}\right)\right]^{2} .
$$

\footnotetext{
${ }^{1}$ UNMSM, Facultad de Ciencias Matemáticas, Lina. Perú. e-mail: vcabanillaszôummsm.edu.pe
} 
Sistemas de la forma (1.1)-(1.2) aparecen en el estudio de la existencia de controles insensitivos para ecuaciones parabólicas semilineales, (ver [2], [3], [8]).

Sea $\Phi: L^{2}(Q) \rightarrow \mathbb{R}$ el funcional diferenciable definido por

$$
\Phi(u)=\frac{1}{2} \int_{0}^{T} \int_{\mathcal{O}} u^{2}(x, t) d x d t
$$

donde $\mathcal{O}$ es llamado conjunto de observación y u es definida sobre el conjunto de soluciones del sistema

$$
\begin{cases}u^{\prime}-\Delta u+f(u, \nabla u)=\xi+h \chi_{\omega} & , \text { en } Q \\ u=0 & , \text { sobre } \Sigma \\ u(x, 0)=y^{0}(x)+\tau u^{0}(x) & , \text { en } \Omega,\end{cases}
$$

En (1.3), $u$ es la función estado y $h$ es la función control. El conjunto $\omega \subset \Omega$ es el conjunto de control, y el término $h \chi_{\omega}$ indica que el control $h$ está actuando únicamente sobre el conjunto $\omega$. La función $f$ es de clase $C^{1}\left(\mathbb{R} \times \mathbb{R}^{n}\right)$, globalmente Lipschitz y $f(0, \overline{0})=0$.

Definición. Decimos que la función $h$ en (1.3) es un control insensitivo de $\Phi$ si

$$
\left.\frac{\partial \Phi(u(x, t ; h, \tau))}{\partial \tau}\right|_{\tau=0}=0, \quad \forall v^{0} \in L^{2}(\Omega)
$$

Esta condición es llamada condición de insensitividad. Esta definición indica que el funcional $\Phi$ es localmente insensitivo ante la perturbación $\tau u^{0}$.

Como fue demostrado en [1], [8], [2], la condición de insensitividad (1.4) es equivalente al problema de controlabilidad exacta para un sistema de ecuaciones parabólicas semilineales de la forma

$$
\begin{gathered}
\begin{cases}y^{\prime}-\Delta y+f(y, \nabla y)=\xi+h \chi_{\omega} & , \text { en } Q \\
y=0 & , \text { sobre } \Sigma \\
y(0)_{\boldsymbol{\Sigma}}=y^{0} & \text { en } \Omega,\end{cases} \\
\begin{cases}-q^{\prime}-\Delta q+f_{\sigma}(y, \nabla y) q-\operatorname{div}\left(f_{\zeta}(y, \nabla y) q\right)=y \chi_{\mathcal{O}} & , \text { en } Q \\
q=0 & , \text { sobre } \Sigma \\
q(T)=0 & , \text { en } \Omega,\end{cases}
\end{gathered}
$$

Por técnicas de punto fijo, el problema de controlabilidad para (1.5)-(1.6) puede ser reducido al problema de la controlabilidad de un sistema linealizado de la forma (1.1)-(1.2).

La principal dificultad cuando se estudia la existencia de controles insensitivos es probar que para algún $M$ suficientemente grande existe $C>0$ tal que la siguiente desigualdad de observabilidad es válida

$$
\int_{0}^{T} \int_{\Omega} \exp \left(-M t^{-1}\right) z^{2} d x d t \leq C \cdot \int_{0}^{T} \int_{\omega} z^{2} d x d t
$$

Para demostrar esta desigualdad es necesaria una estimativa de tipo Carleman para el sistema (1.1)-(1.2).

En este artículo, probaremos una estimativa de tipo Carleman para (1.1)-(1.2), para lo cual usaremos una desigualdad de Carleman debida a O. Yu. Imanuvilov y M. Yamamoto [7] y seguimos las ideas de L. de Teresa [8]. 


\section{Notación y Resultados Previos}

En esta sección damos algunos resultados previos y las notaciones que serán usadas en las siguientes secciones.

Siguiendo [6], introducimos una función auxiliar cuya existencia es demostrada en [6].

Proposición 2.1. Sea $\omega_{0}$ un subconjunto no vacío de $\Omega$. Entonces, existe $\psi=\psi(x) \in C^{2}(\bar{\Omega})$ tal que

$$
\psi>0 \text {, en } \Omega ; \psi=0 \text {, sobre } \partial \Omega ;|\nabla \psi| \neq 0 \text {, en } \overline{\Omega \backslash \omega_{0}} \text {. }
$$

Prueba. Ver [6].

A seguir, introducimos las siguientes funciones

$$
\varphi(x, t)=\frac{e^{\lambda \psi(x)}}{t(T-t)} \quad, \quad \alpha(x, t)=\frac{e^{\lambda \psi(x)}-e^{2 \lambda\|\psi\|_{C(\bar{\Omega})}}}{t(T-t)},
$$

$y$, para $\lambda>1$, definimos los pesos

$$
\eta(x, t)=\frac{e^{\lambda \psi(x)}}{t} \quad \text { y } \quad \tilde{\eta}(x, t)=\frac{e^{\lambda \psi(x)}-e^{2 \lambda\|\psi\|_{C(\bar{\Omega})}}}{t} .
$$

Consideremos el sistema

$$
\begin{cases}y^{\prime}-\Delta y+a y+b . \nabla y=h & , \text { en } Q \\ y=0 & , \text { sobre } \Sigma \\ y(0)=y^{0} & , \text { en } \Omega,\end{cases}
$$

donde $h \in L^{2}\left(0, T ; L^{2}(\Omega)\right)$ y $y^{0} \in L^{2}(\Omega)$. Entonces, la siguiente desigualdad de Carleman es válida.

Proposición $2.2(7)$. Existe un número $\widehat{\lambda}>0$, tal que para un número arbitrario $\lambda \geq \hat{\lambda}$ podemos elegir $s_{0}(\lambda)>0$ tal que: existe una constante $C>0$ tal que para cada $s \geq s_{0}(\lambda)$, la solución $y \in L^{2}\left(0, T ; L^{2}(\Omega)\right)$ del sistema (2.4) satisface la siguiente desigualdad

$$
\begin{aligned}
& \int_{0}^{T} \int_{\Omega}\left(\left(\frac{1}{s \varphi}\right)|\nabla y|^{2}+(s \varphi)|y|^{2}\right) e^{2 s \alpha} d x d t \\
& \leq C\left[\left\|h e^{s \alpha}\right\|_{L^{2}\left(0, T ; H^{-1}(\Omega)\right)}^{2}+\int_{0}^{T} \int_{\omega_{0}}(s \varphi)|y|^{2} e^{2 s \alpha} d x d t\right]
\end{aligned}
$$

Aquí, la constante $C$ depende continuamente de $\lambda,\|a\|_{L^{\infty}(Q)}$ y $\|b\|_{\left[L^{\infty}(Q)\right]^{n}}$. Además, $\omega_{0}$ es el mismo conjunto de (2.1).

6. .

Observación: En adelante, supondremos que $\omega \cap \mathcal{O} \neq \phi$. Sea $r>0$ tal que $B_{r} \subset \omega \cap \mathcal{O}$. Entonces, tomando $\omega_{0}=B_{r}$ en $(2.5)$ tenemos-

$$
\begin{aligned}
& \int_{0}^{T} \int_{\Omega}\left(\left(\frac{1}{s \varphi}\right)|\nabla y|^{2}+(s \varphi)|y|^{2}\right) e^{2 s \alpha} d x d t \\
& \leq C\left[\left\|h e^{s \alpha}\right\|_{L^{2}\left(0, T ; H^{-1}(\Omega)\right)}^{2}+\int_{0}^{T} \int_{B_{r}}(s \varphi)|y|^{2} e^{2 s \alpha} d x d t\right]
\end{aligned}
$$

Notación. Denotemos por $\|$. $\|_{\infty}$ la norma tanto en $L^{\infty}(Q)$ como en $\left[L^{\infty}(Q)\right]^{n}$, y definamos las constantes

$$
\gamma_{1}=\|A\|_{\infty}+\|B\|_{\infty}, \quad \gamma_{2}=\|a\|_{\infty}+\|b\|_{\infty}, \quad \gamma=\gamma_{1}+\gamma_{2}
$$

y

$$
\gamma_{0}=\|a\|_{\infty}^{2}+\|b\|_{\infty}^{2}+\|A\|_{\infty}^{2}+\|B\|_{\infty}^{2}
$$




\section{Resultado Principal}

En esta sección. mostraremos una estimativa de tipo Carleman para la solución $(p, z)$ del sistema (1.1)-(1.2) con potenciales limitados $a, A, b$ y $B$.

Teorema 3.1. Existen constantes $\lambda^{\prime}, s^{\prime}>1$ y $C>0$ tales que para cualquier $\lambda>\lambda^{\prime} y s>s^{\prime}$ la siguiente estimativa es válida para cualquier solución $(p, z)$ de (1.1)-(1.2) con dato inicial $p^{0} \in L^{2}(\Omega)$

$$
\int_{0}^{T} \int_{\Omega}\left(\frac{1}{s \varphi}|\nabla z|^{2}+(s \varphi)|z|^{2}\right) e^{2 s \alpha} d x d t \leq C \int_{0}^{T} \int_{\omega} z^{2} e^{s \alpha} d x d t .
$$

Prueba. Procederemos por etapas.

\section{Etapa 1.}

Aplicando la desigualdad de Carleman (2.6) a la solución $p$ de (1.1), tenemos para $\lambda>\widehat{\lambda} \mathrm{y}$ $s>s_{0}(\lambda)$

$$
\int_{Q}\left(\frac{1}{s \varphi}|\nabla p|^{2}+(s \varphi)|p|^{2}\right) e^{2 s \alpha} d x d t \leq C_{1} \int_{0}^{T} \int_{B_{r}} s \varphi|p|^{2} e^{2 s \alpha} d x d t .
$$

donde $\widehat{\lambda}$ y $s_{0}(\lambda)$ son dadas en la Proposición 2.2.

\section{Etapa 2.}

Sea $r_{1}>r$, tal que $\bar{B}_{r_{1}} \subset \omega \cap \mathcal{O}$, y sea $\xi \in C_{0}^{\infty}(\Omega)$ con

$$
0 \leq \xi \leq 1, \quad \xi(x)=1 \text { si } x \in B_{r}, \quad \xi(x)=0 \text { si } x \in \Omega \backslash B_{r_{1}},
$$

y tal que

$$
\frac{\Delta \xi}{\xi^{1 / 2}} \in L^{\infty}(\Omega) \quad, \quad \frac{\nabla \xi}{\xi^{1 / 2}} \in\left[L^{\infty}(\Omega)\right]^{n} .
$$

Introducimos $u=s \varphi e^{2 s \alpha}$. Entonces, multiplicamos (1.2) por $u \xi p$ en $L^{2}(Q)$, e integrando por partes tenemos

$$
\begin{aligned}
& \int_{Q} z u \xi\left(p^{\prime}-\Delta p+A p+B \cdot \nabla p\right) d x d t+\int_{Q} z u \xi p(a-A) d x d t- \\
& -\int_{Q} z p \Delta(u \xi) d x d t-2 \int_{Q} z \nabla p \cdot \nabla(u \xi) d x d t+\int_{Q} z \xi p u^{\prime} d x d t+ \\
& +\int_{Q} z u \xi(b-B) \cdot \nabla p d x d t+\int_{Q} z p b \cdot \nabla(\xi u) d x d t=\int_{Q} p^{2} u \xi \chi_{\circlearrowleft} d x d t
\end{aligned}
$$

Denotemos las siete integrales de (3.5) por $I_{1}, \ldots, I_{7}$ respectivamente, y estimemos cada una de ellas por separado.

Notemos que $I_{1}=0$ pues $p$ es solución de (1.1). Usando las desigualdades de Hölder y Young tenemos

$$
I_{2}=\int_{Q} z u \xi p(a-A) d x d t \leq \frac{\delta_{1}}{2} \int_{Q} \xi u p^{2} d x d t+\frac{3\left(\|a\|_{\infty}^{2}+\|A\|_{\infty}^{2}\right)}{4 \delta_{1}} \int_{Q} \xi u z^{2} d x d t,
$$

entonces, como $u=s \varphi e^{2 s \alpha}, \xi=0$ en $\Omega \backslash B_{r_{1}}$ y $0 \leq \xi \leq 1$, tenemos

$$
\int_{Q} z u \xi p(a-A) d x d t \leq \frac{\delta_{1}}{2} \int_{Q} \xi u p^{2} d x d t+C \int_{0}^{T} \int_{B_{r_{1}}} s \varphi z^{2} e^{2 s \alpha} d x d t
$$


donde $C$ es una constante que depende de $\|a\|_{\infty}$ y $\|A\|_{\infty}$. Además, $\delta_{1}$ es una constante a ser elegida más adelante.

Estimemos $I_{3}$.

$$
I_{3}=\int_{Q} z p \Delta(u \xi) d x d t
$$

Observemos que

$$
\begin{aligned}
\Delta(u \xi) & =u \Delta \xi+2 \nabla \psi \cdot \nabla \xi\left(s \lambda \varphi+2 s^{2} \lambda \varphi^{2}\right) e^{2 s \alpha}+2 \xi s^{2} \lambda \varphi^{2}(\Delta \psi) e^{2 s \alpha}+ \\
& +\xi\left(s \lambda^{2} \varphi+6 s^{2} \lambda^{2} \varphi^{2}+4 s^{3} \lambda^{2} \varphi^{3}\right)|\nabla \psi|^{2} e^{2 s \alpha}
\end{aligned}
$$

entonces

$$
\begin{gathered}
I_{3}=\int_{Q} z p u \Delta \xi d x d t+2 \int_{Q} z p \nabla \psi \cdot \nabla \xi\left(s \lambda \varphi+2 s^{2} \lambda \varphi^{2}\right) e^{2 s \alpha} d x d t+ \\
+2 \int_{Q} z p \xi s^{2} \lambda \varphi^{2}(\Delta \psi) e^{2 s \alpha} d x d t+\int_{Q} z p \xi\left(s \lambda^{2} \varphi+6 s^{2} \lambda^{2} \varphi^{2}+4 s^{3} \lambda^{2} \varphi^{3}\right)|\nabla \psi|^{2} e^{2 s \alpha} d x d t .
\end{gathered}
$$

Escribamos (3.8) como $I_{3}=J_{1}+J_{2}+J_{3}+J_{4}$. Para estimar cada $J_{k}$ agrupamos los coeficientes de $z$ y $p$. Como $\xi=0$ en $\Omega \backslash B_{r_{1}}$, usando desigualdades elementales tenemos que

$$
\begin{gathered}
J_{1} \leq \frac{\delta_{2}}{2} \int_{0}^{T} \int_{B_{r_{1}}} \xi p^{2} u d x d t+C \int_{0}^{T} \int_{B_{r_{1}}} z^{2} u d x d t \\
J_{2} \leq \frac{\delta_{3}}{2} \int_{0}^{T} \int_{B_{r_{1}}} \xi p^{2} u d x d t+C \int_{0}^{T} \int_{B_{r_{1}}} s^{2} \lambda^{2} \varphi^{2} z^{2} u d x d t . \\
J_{3} \leq \frac{\delta_{4}}{2} \int_{0}^{T} \int_{B_{r_{1}}} \xi p^{2} u d x d t+C \int_{0}^{T} \int_{B_{r_{1}}} s^{2} \lambda^{2} \varphi^{2} u z^{2} d x d t . \\
J_{4} \leq \frac{\delta_{5}}{2} \int_{0}^{T} \int_{B_{r_{1}}} \xi p^{2} u d x d t+C \int_{0}^{T} \int_{B_{r_{1}}} \lambda^{4} s^{4} \varphi^{4} z^{2} u d x d t .
\end{gathered}
$$

Reemplazando (3.9), (3.10), (3.11) y (3.12) en (3.8) tenemos, para $\lambda \geq \lambda_{2}$

$$
I_{3} \leq \frac{\sum_{2 \leq i \leq 5} \delta_{i}}{2} \int_{0}^{T} \int_{B_{r_{1}}} \xi p^{2} u d x d t+C \int_{0}^{T} \int_{B_{r_{1}}} \lambda^{4} s^{4} \varphi^{4} z^{2} u d x d t
$$

y siendo $u=s \varphi e^{2 s \alpha}$ tenemos

$$
I_{3} \leq \frac{\sum_{2 \leq i \leq 5} \delta_{i}}{2} \int_{0}^{T} \int_{B_{r_{1}}} \xi p^{2} u d x d t+C \int_{0}^{T} \int_{B_{r_{1}}} \lambda^{4} s^{5} \varphi^{5} z^{2} e^{2 \xi \alpha} d x d t
$$

Con el fin de estimar $I_{4}$ agrupamos los coeficientes de $z$ y $\nabla p$ para obtener

$$
I_{4} \leq \frac{\beta_{1}}{2} \int_{0}^{T} \int_{\Omega} \frac{u \xi|\nabla p|^{2}}{\lambda(s \varphi)^{2}} d x d t+C \int_{0}^{T} \int_{B_{r_{1}}} \lambda^{3} s^{5} \varphi^{5} z^{2} e^{2 s \alpha} d x d t
$$

Para $I_{5}$, observamos que

$$
u^{\prime}=s \varphi^{\prime} e^{2 s \alpha}+2 s^{2} \varphi e^{2 s \alpha} \alpha^{\prime}
$$


y $\left|\varphi^{\prime}\right| \leq C(T) \varphi^{2}$. Siguiendo los argumentos anteriores llegamos a

$$
I_{5} \leq \frac{\delta_{6}}{2} \int_{0}^{T} \int_{B_{r_{1}}} \xi p^{2} u d x d t+C \int_{0}^{T} \int_{B_{r_{1}}} s^{3} \varphi^{5} z^{2} e^{2 s \alpha} d x d t
$$

Análogamente

$$
I_{6} \leq \frac{\beta_{2}}{2} \int_{0}^{T} \int_{\Omega} \frac{1}{\lambda(s \varphi)^{2}} \xi|\nabla p|^{2} u d x d t+C \int_{0}^{T} \int_{B_{r_{1}}} \lambda s^{3} \varphi^{3} z^{2} e^{2 s \alpha} d x d t
$$

y

$$
I_{7} \leq \frac{\delta_{7}}{2} \int_{0}^{T} \int_{B_{r_{1}}} \xi p^{2} u d x d t+C \int_{0}^{T} \int_{B_{r_{1}}} s^{3} \lambda^{2} \varphi^{3} z^{2} e^{2 s \alpha} d x d t
$$

Reemplazando (3.6), (3.7), (3.13), (3.14), (3.15), (3.16) y (3.17) en (3.5) tenemos

$$
\begin{gathered}
\int_{0}^{T} \int_{B_{r_{1}}} \xi p^{2} u d x d t \leq \frac{\sum_{1 \leq i \leq 7} \delta_{i}}{2} \int_{0}^{T} \int_{B_{r_{1}}} \xi p^{2} u d x d t+ \\
+\frac{\beta_{1}+\beta_{2}}{2} \int_{0}^{T} \int_{\Omega} \frac{\xi|\nabla p|^{2} u}{\lambda(s \varphi)^{2}} d x d t+C\left(1+\gamma_{0}\right) \int_{0}^{T} \int_{B_{r_{1}}} s^{5} \lambda^{4} \varphi^{5} z^{2} e^{2 s \alpha} d x d t
\end{gathered}
$$

Ahora, eligiendo $\delta_{i}>0$ y $\beta_{i}>0$ tales que $\sum_{1 \leq i \leq 7} \delta_{i}=\beta_{1}+\beta_{2}=1$ concluimos de (3.18) que

$$
\int_{0}^{T} \int_{B_{r_{1}}} \xi p^{2} u d x d t \leq \int_{0}^{T} \int_{\Omega} \frac{\xi|\nabla p|^{2} u}{\lambda(s \varphi)^{2}} d x d t+C\left(1+\gamma_{0}\right) \int_{0}^{T} \int_{B_{r_{1}}} \lambda^{4} s^{5} \varphi^{5} z^{2} e^{2 s \alpha} d x d t
$$

siendo $\gamma_{0}=\|A\|_{\infty}^{2}+\|B\|_{\infty}^{2}+\|a\|_{\infty}^{2}+\|b\|_{\infty}^{2}$.

Reemplazando $u=s \varphi e^{2 s \alpha}$ en (3.19) y como $\xi=0$ sobre $\Omega \backslash B_{r_{1}}$, y $\xi(x)=1$ si $x \in B_{r}$, llegamos a

$$
\begin{gathered}
\int_{0}^{T} \int_{B_{r}} s \varphi p^{2} e^{2 s \alpha} d x d t \leq \int_{0}^{T} \int_{B_{r_{1}}} \frac{1}{\lambda s \varphi}|\nabla p|^{2} e^{2 s \alpha} d x d t+ \\
\quad+C\left(1+\gamma_{0}\right) \int_{0}^{T} \int_{B_{r_{1}}} \lambda^{4} s^{5} \varphi^{5} z^{2} e^{2 s \alpha} d x d t
\end{gathered}
$$

Aplicando (3.20) en (3.2) obtenemos

$$
\begin{gathered}
\int_{0}^{T} \int_{\Omega}\left(\frac{1}{s \varphi}|\nabla p|^{2}+(s \varphi)|p|^{2}\right) e^{2 s \alpha} d x d t \leq C_{1}\left[\int_{0}^{T} \int_{B_{r_{1}}} \frac{|\nabla p|^{2}}{\lambda s \varphi} e^{2 s \alpha} d x d t+\right. \\
\left.+C\left(1+\gamma_{0}\right) \int_{0}^{T} \int_{B_{r_{1}}} \lambda^{4} s^{5} \varphi^{5} z^{2} e^{2 s \alpha} d x d t\right]
\end{gathered}
$$

Entonces

$$
\begin{gathered}
\left(1-\frac{C_{1}}{\lambda}\right) \int_{0}^{T} \int_{\Omega} \frac{1}{s \varphi}|\nabla p|^{2} e^{2 s \alpha} d x d t+\int_{0}^{T} \int_{\Omega}(s \varphi)|p|^{2} e^{2 s \alpha} d x d t \leq \\
\leq C\left(1+\gamma_{0}\right) \int_{0}^{T} \int_{B_{r_{1}}} s^{5} \lambda^{4} \varphi^{5} z^{2} e^{2 s \alpha} d x d t
\end{gathered}
$$


En consecuencia, para $\lambda \geq \lambda_{3}=\operatorname{máx}\left\{\lambda_{2}, C_{1}\right\}$ tenemos, para cualquier $\lambda \geq \lambda_{3}$ y $s \geq s(\lambda)$

$$
\int_{0}^{T} \int_{\Omega}\left(\frac{1}{s \varphi}|\nabla p|^{2}+(s \varphi)|p|^{2}\right) e^{2 s \alpha} d x d t \leq C\left(1+\gamma_{0}\right) \int_{0}^{T} \int_{B_{r_{1}}} \lambda^{4} s^{5} \varphi^{5} z^{2} e^{2 s \alpha} d x d t .
$$

\section{Etapa 3.}

Aplicando la desigualdad de Carleman (2.5) a la solución $z$ de (1.2) tenemos

$$
\begin{gathered}
\int_{0}^{T} \int_{\Omega}\left(\frac{1}{s \varphi}|\nabla z|^{2}+(s \varphi)|z|^{2}\right) e^{2 s \alpha} d x d t \leq \\
\leq C_{1}\left(\left\|p \chi_{\circlearrowleft} e^{s \alpha}\right\|_{L^{2}\left(0, T ; H^{-1}(\Omega)\right)}^{2}+\int_{0}^{T} \int_{B_{r_{1}}}(s \varphi) z^{2} e^{2 s \alpha} d x d t\right) .
\end{gathered}
$$

Por la continuidad de la inmersión $L^{2}\left(0, T ; L^{2}(\Omega)\right) \hookrightarrow L^{2}\left(0, T ; H^{-1}(\Omega)\right)$ se tiene

$$
\begin{gathered}
\int_{0}^{T} \int_{\Omega}\left(\frac{1}{s \varphi}|\nabla z|^{2}+(s \varphi)|z|^{2}\right) e^{2 s \alpha} d x d t \leq \\
\leq C\left(\int_{0}^{T} \int_{\mathcal{O}}(s \varphi) p^{2} e^{2 s \alpha} d x d t+\int_{0}^{T} \int_{B_{r_{1}}}(s \varphi) z^{2} e^{2 s \alpha} d x d t\right) .
\end{gathered}
$$

Aplicando la desigualdad (3.22) en (3.24) concluimos que

$$
\begin{gathered}
\int_{0}^{T} \int_{\Omega}\left(\frac{1}{s \varphi}|\nabla z|^{2}+(s \varphi)|z|^{2}\right) e^{2 s \alpha} d x d t \leq \\
\leq C\left(1+\gamma_{0}\right) \int_{0}^{T} \int_{B_{r_{1}}} \lambda^{4} s^{5} \varphi^{5} z^{2} e^{2 s \alpha} d x d t+C \int_{0}^{T} \int_{B_{r_{1}}}(s \varphi) z^{2} e^{2 s \alpha} d x d t .
\end{gathered}
$$

Entonces, la desigualdad (3.1) es una consecuencia inmediata de (3.25) y el siguiente resultado técnico.

Lema 3.1. Sea $\lambda \geq \lambda_{3}$, entonces existe $s^{\prime}=s^{\prime}(\lambda)>1$ tal que para $s>s^{\prime}$ se tiene

$$
\lambda^{4} s^{5} \varphi^{5} e^{s \alpha} \leq 1 \quad y \quad s \varphi e^{s \alpha} \leq 1
$$

Agradecimiento. El autor desea agradecer al Profesor L. A. Medeiros por las conversaciones que tuvimos en relación al presente trabajo.

\section{REFERENCES}

[1] O. BODART y C. FABRE, Controls insensitizing the norm of the solution of a semilinear heat equation. J. Math. Anal. and Appl., 195, (1995), pp. 658-683.

[2] V. CABANILLAS, Insensitizing Controls for a Semilinear Heat Equation Involving Gradient Terms, Proceeding of $53^{0}$ SBA, UEM, 2001.

[3] V. CABANILLAS, Insensitizing Controls for a Semilinear. Heat Equation in Unbounded Domains, Proceeding of $54^{0}$ SBA, UNESP, 2001.

[4] V. CABANILLAS, S. MENEZES y E. ZUAZUA, Null controllability in unbounded domains for the semilinear heat equations with nonlinearities involving gradient terms., J. Optim. Theory Applications, 110. (2) (2001). 
[5] C. FABRE, J. P. PUEL y E. ZUAZUA, Approximate controllability of the semilinear heat equation. Proc. Royal Soc. Edinburgh, 125A, (1995), pp. 31-61.

[6] A. FURSIKOV y O. Yu. IMANUVILOV, Controllability of evolution equations. Lecture Notes Series \#34. Research Institute of Mathematics, Global Analysis Research Center, Seoul National University, (1996).

[7] O. Yu. IMANUVILOV e M. YAMAMOTO, On Carleman inequalities for parabolic equations in Sobolev spaces of negative order and exact controllability for semilinear parabolic equations. Preprint \# 98-46, University of Tokyo. Graduate School of Mathematics, Tokyo, Japan, (1998).

[8] L. de TERESA, Insensitizing controls for a semilinear heat equation. Comm. in Partial Diff. Equations 25 (1832), (2000), pp 39-72.

[9] E. ZUAZUA, Finite dimensional null controllability for the semilinear heat equation. $J$. Math. Pures Appl., 76, (1997), pp. 237-264. 